\title{
Acute ischemic stroke on the setting of left atrial myxoma: optimal time for cardiac surgery
}

Quoc Bui ${ }^{1}$, San $\operatorname{Tran}^{2}$, Steven Pham ${ }^{3}$, Vien Phan ${ }^{4}$, Khiet Nguyen ${ }^{5}$, Ngoc Mai ${ }^{6}$, Thoai Nguyen ${ }^{5}$, Lac Nguyen ${ }^{5}$, Thanh Hoang ${ }^{7}$, Chuong Le ${ }^{5}$, Hashim Chaudhry ${ }^{8}$, Amandeep Goyal ${ }^{9}$, Duc Nguyen ${ }^{5}$, Dat Q Ha ${ }^{10}$, Doi Huynh ${ }^{11}$, Phillip Tran*12

${ }^{1}$ Division of Biostatistics, Washington University, School of Medicine in St. Louis, St Louis, MO, USA

${ }^{2}$ PGY2 - Department of Internal medicine, Mount Sinai Hospital Medical Center of Chicago, Chicago City, IL, USA

${ }^{3}$ Kansas City University College of Osteopathic Medicine, Kansas City, MO, USA

${ }^{4}$ Kansai Medical University, Japan

${ }^{5}$ University of Medicine and Pharmacy HCM City, Vietnam

${ }^{6}$ Pham Ngoc Thach University of Medicine, Vietnam

${ }^{7}$ Vietnam Military Medicine University, Vietnam

${ }^{8}$ AT Still School of Osteopathic Medicine, Mesa, AZ, USA

${ }^{9}$ University of Kansas Medical Center, Kansas, KS, USA

${ }^{10}$ St Rose Hospital, Hayward, USA

${ }^{11}$ Nam Can Tho University, Vietnam

${ }^{12}$ Yavapai Regional Medical Center, Prescott, AZ, USA

Received: July 4, 2021

DOI: $10.5430 /$ crim.v8n2p1
Accepted: September 13, 2021

Online Published: September 23, 2021

\begin{abstract}
The optimal time of left atrial (LA) myxoma resection is still controversial due to the risk of embolic phenomena of delayed surgery versus the risk of hemorrhagic transformation of early surgical intervention. Here, we describe a case of 60-year-old man without significant past medical history admitted with two-day of double vision and found to have LA myxoma. Multiple factors including clinical characteristics and comorbidities should be considered to decide the favorable management.
\end{abstract}

Key Words: Cardiac myxoma, Ischemic stroke, Neurologic deficit, Surgical excision

\section{INTRODUCTION}

Cardiac myxoma is the most common cause of primary cardiac tumor and is responsible for $0.5 \%$ of stroke. Cardiac myxoma can present from nonspecific symptoms to critical presentation. Appropriate management of cardiac myxoma is still controversial due to the concern of neurologic deterioration. Different clinical factors, such as time of onset, level of neurologic deficit, risk of recurrence, size of the tumor, underlying comorbidities as well as bleeding risk should be evaluated to determine the timing of surgery.

\section{CASE PRESentation}

A 60-year-old-man without significant past medical history was admitted to the hospital for two-day of diplopia. He reported no cardiovascular or neurologic symptoms such as chest pain, palpitation, dyspnea, numbness, or weakness. Vi-

\footnotetext{
*Correspondence: Phillip Tran; Email: ptrannyit@gmail.com; Address: Dignity Health Yavapai Regional Medical Center, 726 Gail Gardner Way, Prescott, AZ 86301, Vietnam.
} 
tal signs showed a heart rate of 89 beats per minute, arterial blood pressure of $129 / 82 \mathrm{mmHg}$, a blood oxygen saturation of $98 \%$ on room air, and a respiratory rate of 16 per minute. Heart rate and rhythm were regular. Non-radiating $2 / 6$ holosystolic murmur was best heard at the apex. The patient was oriented to person, place, and time. No movements or sensations dysfunction was found. Based on the clinical presentations above, the differential diagnoses included atherosclerotic stroke, carotid stenosis with complicated stroke, and embolic stroke with cardiac origin.

In the emergency department, head CT-scan without contrast showed hypoattenuating areas in the left thalamus, right frontal, and parietal subcortical white matter favoring infarcts of unknown acuity (see Figure 1). Brain MRI confirmed a small acute left thalamic infarct with advanced microvascular ischemic changes (see Figure 2). Transthoracic echocardiogram showed a large mobile, pedunculated mass measure $2.34 \mathrm{~cm} \times 2.19 \mathrm{~cm}$ (see Figure 3). Following transesophageal echocardiogram also confirmed a large LA mass with the size $2.88 \mathrm{~cm} \times 1.91 \mathrm{~cm}$ in the LA that inserted to the lower portion of the interatrial septum. No patent foramen ovale, mitral valve inflow obstruction, or evidence of thrombosis were found. Carotid doppler ultrasound also showed no significant findings. The imaging appearance highly suggested that LA myxoma could be a possible cause of acute ischemic stroke.

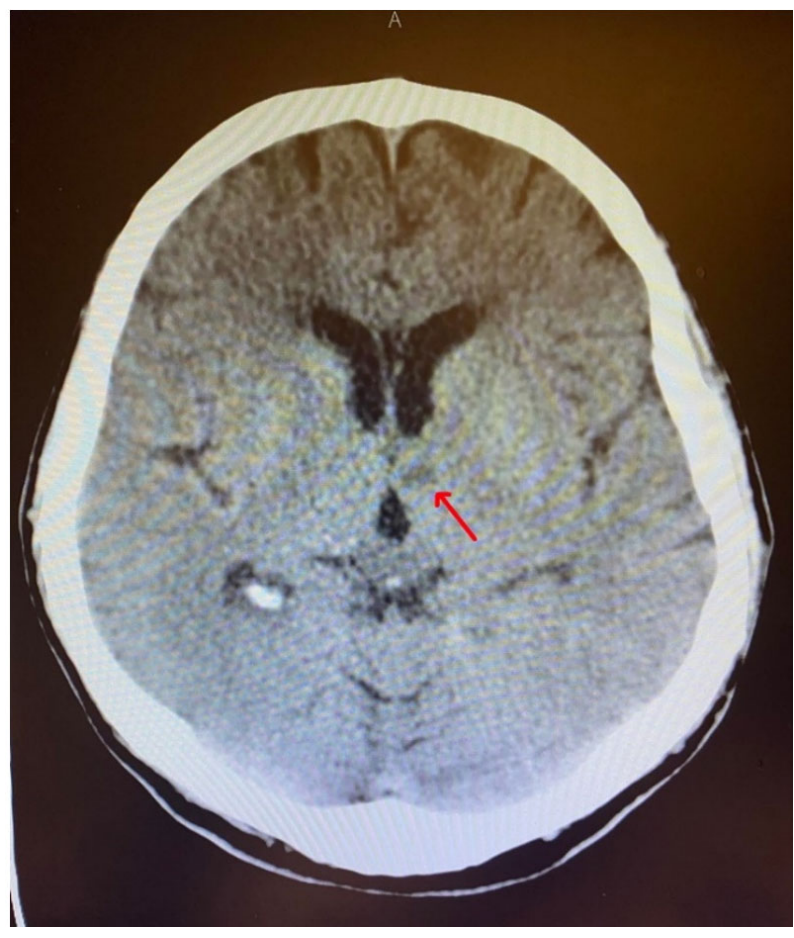

Figure 1. CT head - hypoattenuating areas in the left thalamus, right frontal, and parietal subcortical white matter favoring infarcts of unknown acuity

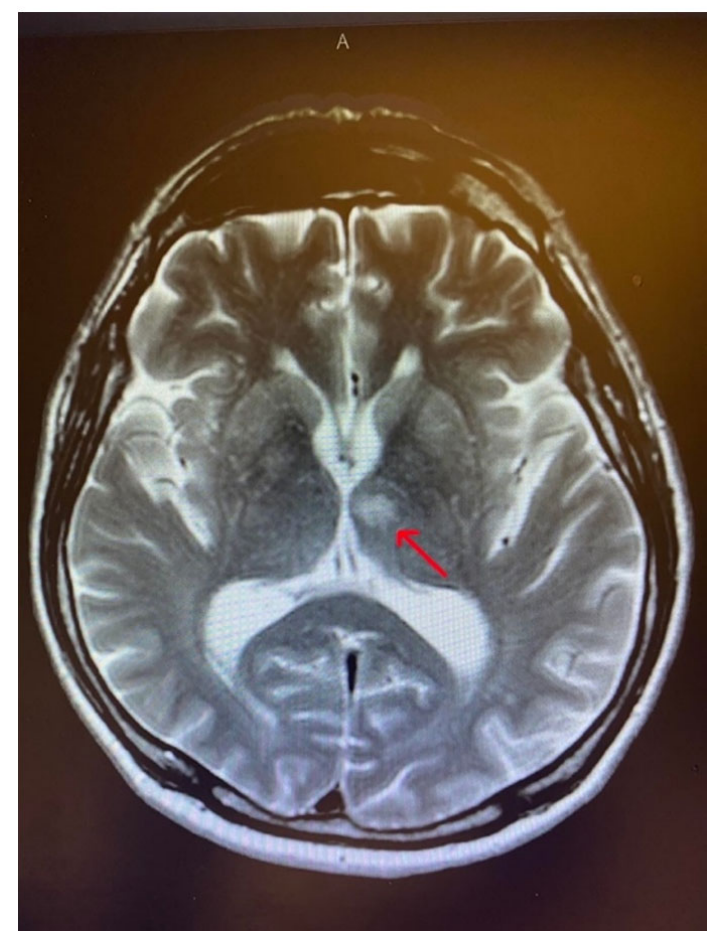

Figure 2. Brain MRI - small acute left thalamic infarct with advanced microvascular ischemic changes

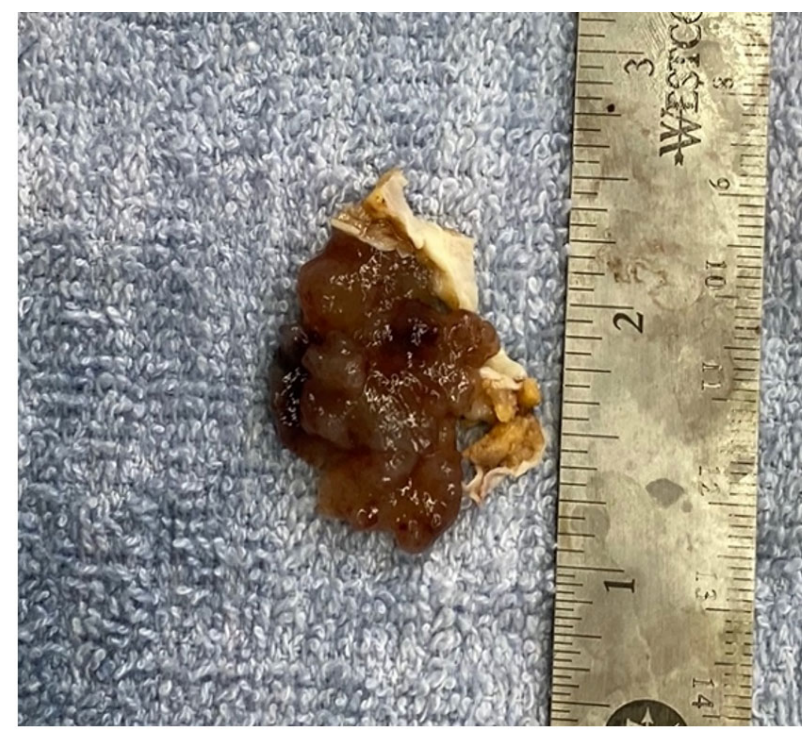

Figure 3. Pedunculated mass measure $23.4 \mathrm{~mm} \times 21.9 \mathrm{~mm}$

Thoracic cardiac surgery was promptly consulted due to the high risk of further embolization and low risk of intracerebral hemorrhage. LA mass excision was indicated. No significant coronary arterial diseases were found on coronary angiograms. The patient underwent successful open-heart surgery with excision of left atrial myxoma without hemorrhagic event. On initial gross anatomy evaluation, a large gelatinous lesion $20 \mathrm{~mm} \times 30 \mathrm{~mm}$ attached to the interatrial septum on the LA was removed. The result of histopathology 
also confirmed cardiac myxoma (see Figure 4). The patient was discharged seven days after the surgery. The cardiac and neurologic symptoms were routinely evaluated on the follow- up visits. His neurologic deficit was rapidly improving, and no hemorrhagic events were found.

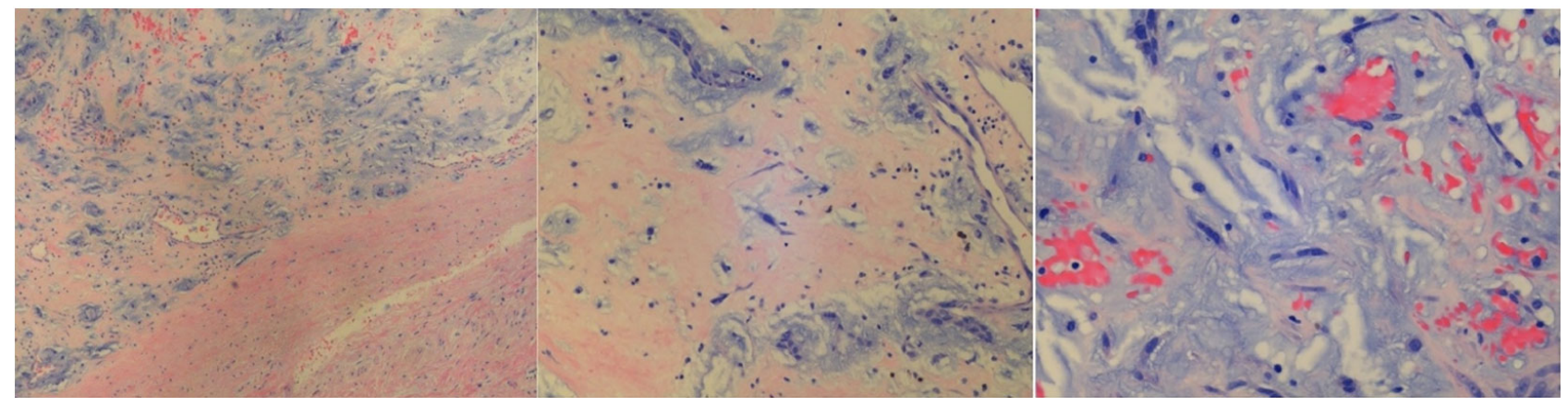

Figure 4. Histopathology also confirmed the cardiac myxoma

\section{Discussion}

Cardiac myxoma is the most common primary cardiac neoplasm diagnosed in adults, which accounts for up to $80 \%$ of benign tumors. Most common locations of myxomas include left atrium, $60 \%$ to $80 \%$ right atrium (25\%), or ventricle $(12 \%) .{ }^{[1]}$ The average size of atrial myxoma is $27 \mathrm{~mm}$ with the range from 4 to $65 \mathrm{~mm} \cdot{ }^{[2,3]}$ The clinical manifestation of cardiac myxoma is non-specific and depends on the location, size, and mobility of the tumor. The classic triad of cardiac myxoma includes obstructive (syncope, arrhythmia, palpitation, and sudden cardiac death), embolic and constitutional symptoms (fever, weight loss, arthralgia, and fatigue). Once the diagnosis has been confirmed by imaging, prompt tumor resection is required following routine preoperative evaluation because of the risk of embolization or cardiovascular complications. The outcome of the surgery is generally good with the mortality rate under $5 \% .{ }^{[4]}$

Among those complications of cardiac myxoma, embolic event has been occurred in up to $30 \%-50 \%$, accounted for $0.5 \%$ cause of stroke. ${ }^{[1]}$ The risk of recurrence also occurs in the range from $1 \%$ to $3 \%$ of sporadic case, but it may increase up to $12 \%$ in some congenital conditions, such as Carney complex myxoma. ${ }^{[5]}$

Clinical manifestation of cardiac myxoma stroke is usually of acute onset and women at the age of 50 years carry the greatest risk of developing cardiac myxoma. ${ }^{[5]}$ Other consequences of tumor embolization including metastasis and myxoma induced aneurysm also present in the delayed progress of the disease. Also, cerebrovascular events typically present as embolic ischemic and rarely as hemorrhagic events. ${ }^{[2]}$ Some retrospective studies and case series also show that embolic event in myxoma usually occurs at multiple sites, such as cerebral arteries and peripheral or retinal arteries. ${ }^{[6]}$

Currently, there is no guideline for the management of cardiac myxoma related stroke due to limited number of randomized trials and prospective studies. Although the curative treatment of cardiac myxoma is surgical excision, the optimal time after cardiovascular embolism (CVE) is still controversial. Some authors suggest emergency surgery after the diagnosis confirmed to prevent further embolization. ${ }^{[7]}$ The others suggest delay surgical removal due to the risk of hemorrhagic transformation possibly caused by high dose anticoagulant during emergency surgery. ${ }^{[8,9]}$ Thus, an alternative strategy has been suggested by using antiplatelet or anticoagulant for CVE and delay the surgery 4-6 weeks after the event, especially in the case of tumors less than 20 $\mathrm{mm} .^{[10]}$

In our case, several clinical factors were considered for surgical decision. First, the patient had a low risk of atherosclerosis disease, good functional capacity, small ischemic lesion, and less bleeding risk. Those factors were considered a low risk of hemorrhagic transformation in this case. Second, an initial transesophageal echocardiogram showed $28 \mathrm{~mm}$, pedunculated, mobile mass in the LA made this tumor has a high risk of complication, including obstruction, embolic, and recurrence. Therefore, emergency cardiac excision was reasonable at that time. After the surgery, the patient was discharged without hemorrhagic events. His cardiac and neurologic status was improving on the following routine visit.

\section{CONFlicts of InTEREST Disclosure}

The authors have declared no conflicts of interest. 


\section{REFERENCES}

[1] Gosev I, Paic F, Duric Z, et al. Cardiac myxoma the great imitators: comprehensive histopathological and molecular approach. Int J Cardiol. 2013; 164(1): 7-20. PMid:22243936. https://doi .org/10 $.1016 / j . i j c a r d .2011 .12 .052$

[2] Lee VH, Connolly HM, Brown RD, Jr. Central nervous system manifestations of cardiac myxoma. Archives of neurology. 2007; 64(8): 1115-20. PMid:17698701. https://doi.org/10.1001/archne ur.64.8.1115

[3] Lee SJ, Kim JH, Na CY, et al. Eleven years' experience with Korean cardiac myxoma patients: focus on embolic complications. Cerebrovasc Dis. 2012; 33(5): 471-9. PMid:22517375. https: //doi.org/10.1159/000335830

[4] Keeling IM, Oberwalder P, Anelli-Monti M, et al. Cardiac myxomas: 24 years of experience in 49 patients. Eur J Cardiothorac Surg. 2002; 22(6): 971-7. https : //doi .org/10.1016/S1010-7 940 (02) 00592-4

[5] Waller DA, Ettles DF, Saunders NR, et al. Recurrent cardiac myxoma: the surgical implications of two distinct groups of patients.
The Thoracic and Cardiovascular Surgeon. 1989; 37(4): 226-30. PMid:2799794. https://doi .org/10.1055/s-2007-1020322

[6] Karabinis A, Samanidis G, Khoury M, et al. Clinical presentation and treatment of cardiac myxoma in 153 patients. Medicine (Baltimore). 2018; 97(37): e12397.PMid:30213011. https://doi.org/10.1 097/MD.0000000000012397

[7] Al-Said Y, Al-Rached H, Baeesa S, et al. Emergency excision of cardiac myxoma and endovascular coiling of intracranial aneurysm after cerebral infarction. Case Rep Neurol Med. 2013; 2013: 839270. PMid:24109531. https://doi .org/10.1155/2013/839270

[8] Zisbrod Z, Rose DM, Jacobowitz IJ, et al. Results of open heart surgery in patients with recent cardiogenic embolic stroke and central nervous system dysfunction. Circulation. 1987; 76(5 Pt 2): V109-12.

[9] Ekinci EI, Donnan GA. Neurological manifestations of cardiac myxoma: a review of the literature and report of cases. Intern Med J. 2004; 34(5): 243-9. PMid:15151670. https://doi .org/10.111 1/j.1444-0903.2004.00563.x

[10] Badui Dergal E, Cordero E, Soberanis N, et al. [Cardiac myxoma. A report of 23 cases]. Gac Med Mex. 1992; 128(3): 245-52. 